\title{
UNDISCOUNTED MARKOV CHAIN BSDES TO STOPPING TIMES
}

\author{
SAMUEL N. COHEN, ${ }^{*}$ University of Oxford
}

\begin{abstract}
We consider backward stochastic differential equations in a setting where noise is generated by a countable state, continuous time Markov chain, and the terminal value is prescribed at a stopping time. We show that, given sufficient integrability of the stopping time and a growth bound on the terminal value and BSDE driver, these equations admit unique solutions satisfying the same growth bound (up to multiplication by a constant). This holds without assuming that the driver is monotone in $y$, that is, our results do not require that the terminal value be discounted at some uniform rate. We show that the conditions are satisfied for hitting times of states of the chain, and hence present some novel applications of the theory of these BSDEs.
\end{abstract}

Keywords: BSDE; Markov chain; uniform ergodicity; risk averse control; non-Ohmic circuit

2010 Mathematics Subject Classification: Primary 60J27

Secondary 93E20; 94C05

\section{Introduction}

Since their early introduction by Bismut [1] (in the linear case) and, in particular, since the nonlinear existence result of Pardoux and Peng [19], backward stochastic differential equations (BSDEs) have risen to be a powerful component of the stochastic analyst's toolkit. The early conditions of the theory (i.e. that the driver of the BSDE be Lipschitz, that the terminal time is deterministic, and that the filtration is generated by a Brownian motion) have all been extended, leaving a rich class of equations which can be used in many practical problems. In particular, for our discussion here, the theory of BSDEs when noise is driven by a finite or countable state Markov chain (in continuous time) has been developed by the author and collaborators in a series of recent papers; see [4], [5], [7], and [8].

In this paper, we consider a novel extension of the theory of BSDEs, when the terminal time is replaced by an unbounded stopping time. We say that this is a novel extension; however, previous work has approached this question in the Brownian setting (see, in particular, [2], [12], and [21]. In those papers, a key assumption of monotonicity of the driver is made, essentially corresponding to discounting the future at a rate which is bounded away from zero. This allows these authors to show that the problem is well-posed, in particular, that the BSDE admits unique solutions, and that these solutions can be well approximated using finite-time BSDEs. A related setting is discussed in [7, Section 2] in the Markov chain case, again with an assumption of a discounting term.

In this paper, we approach the problem somewhat differently. We seek to show that we can impose conditions on the stopping time directly such that the BSDE admits unique solutions

Received 22 February 2013; revision received 8 May 2013.

* Postal address: Mathematical Institute, University of Oxford, 24-29 St Giles', Oxford OX1 3LB, UK.

Email address: samuel.cohen@maths.ox.ac.uk 
(at least, unique solutions satisfying some integrability conditions), and that these conditions can be verified, for example, when the stopping time is a hitting time. In this case, our conditions can be connected to the uniform ergodicity of the Markov chain under a family of measures. These questions of ergodicity were explored in [7] in the context of proving the existence of ergodic BSDE solutions, and we draw liberally on these results. In all our analysis, we do not assume the monotonicity of the driver, which in particular allows us to consider the case when the driver depends only on the $Z$ component of the solution, that is, when the BSDE is effectively performing a nonlinear change of measure.

We then present a couple of applications, both the standard application of BSDEs to control problems, and a novel application to our understanding of non-Ohmic electronic circuits, by relating these to a Markov chain model.

The paper first presents the basic theory of BSDEs on Markov chains (Section 1.1), then gives our existence proof (Section 2), then considers how the conditions of the existence proof could be verified for hitting times (Section 3), and ends with applications (Section 4).

\subsection{Introducing BSDEs on Markov chains}

Consider a continuous-time, countable state process $X$ in a probability space $(\Omega, \mathcal{F}, \mathbb{P})$. We shall suppose that $X$ is a Markov chain in its own filtration, under the measure $\mathbb{P}$. Without loss of generality, we shall represent $X$ as taking values from the standard basis vectors $e_{i}$ of $\mathbb{R}^{N}$ (where $N \in \mathbb{N} \cup\{\infty\}$ is the number of states, and $\mathbb{R}^{\infty}$ denotes the space of infinite real sequences). We write $\mathcal{X}$ for this set of basis vectors. For notational simplicity, we will think of all vectors as column vectors, and denote by $z^{\top}$ the transpose of $z$ (so that $z^{\top} y$ is the Euclidean or $\ell_{2}$ inner product, and $e_{i}^{\top} z$ is the $i$ th component of $z$ ). An element $\omega \in \Omega$ can be thought of as describing a path of the chain $X$.

Let $\left\{\mathcal{F}_{t}\right\}_{t \geq 0}$ be the completion of the filtration generated by $X$, that is

$$
\mathcal{F}_{t}=\sigma\left(\left\{X_{s}\right\}_{s \leq t}\right) \vee\left\{B \in \mathcal{F}_{\infty}: \mathbb{P}(B)=0\right\} .
$$

As $X$ is a right-continuous finite activity pure jump process which does not jump at time 0 , this filtration is right-continuous, and we assume that $\mathcal{F}=\mathscr{F}_{\infty}=\bigvee_{t<\infty} \mathcal{F}_{t}$. For the basic theory of continuous-time countable-state Markov chains see, for example, [20, p. $228 \mathrm{ff}]$; for the approach taken here see [14, Part III].

Let $A$ denote the possibly infinite rate matrix of the chain $X$. (In our notation, as in [14], $A$ is the matrix with entries $A_{i j}$, where $A_{i j}$ is the rate of jumping from state $j$ to state $i$. Depending on the convention used, this is either the rate matrix or its transpose. In our notation, $A^{\top}$, the transpose of $A$, is the generator of the Markov chain.) Note that $\left(A_{t}\right)_{i j} \geq 0$ for $i \neq j$ and $\sum_{i}\left(A_{t}\right)_{i j}=0$ for all $j$ (the columns of $A$ all sum to 0 ). We assume, for simplicity, that the entries in $A_{t}$ are uniformly bounded, and so the chain is regular.

From the Doob-Meyer decomposition (see [14, Appendix B]), we write our chain in the following way:

$$
X_{t}=X_{0}+\int_{] 0, t]} A_{u} X_{u-} \mathrm{d} u+M_{t},
$$

where $M$ is a locally-finite-variation pure-jump $\mathbb{P}$-martingale in $\mathbb{R}^{N}$, and the chain starts in state $X_{0} \in \mathcal{X}$. Our aim is to study a class of BSDEs up to stopping times, that is, equations of the form

$$
Y_{t}=\xi+\int_{] t, \tau]} f\left(\omega, u, Y_{u-}, Z_{u}\right) \mathrm{d} u-\int_{] t, \tau]} Z_{u}^{\top} \mathrm{d} M_{u}, \quad 0 \leq t \leq \tau<\infty,
$$


where $\tau$ is an integrable stopping time, $f: \Omega \times \mathbb{R}^{+} \times \mathbb{R} \times \mathbb{R}^{N} \rightarrow \mathbb{R}$ is a given function, $Y$ is a real-valued càdlàg stochastic process, $Z$ is a predictable process in $\mathbb{R}^{N}$ such that

$$
\int_{] 0, t]} Z_{u}^{\top} \mathrm{d} M_{u}:=\sum_{i} \int_{] 0, t]}\left(Z_{u}\right)^{i} \mathrm{~d}(M)_{u}^{i}
$$

is a square-integrable martingale (here $(\cdot)^{i}$ denotes the $i$ th component of the vector), and $\xi$ is $\mathcal{F}_{\tau}$-measurable. The key problem is that we do not assume that $\tau$ is uniformly bounded, nor that $f$ has monotone dependence on $Y$. Such assumptions are needed to apply the 'discounted' BSDE methods in [7] or [2].

Remark 1. We note that the use of left limits for $Y$ in the driver term of (1) initially seems unconventional, for those used to the theory of BSDEs in a Brownian setting. However, it is the natural approach when the driver term can itself jump (see [6]), it ensures that the driver is predictable, and as the integral is with respect to Lebesgue measure and our processes have at most countably many jumps, in this case the equation is unchanged whether the left limits are included or not.

The following process and the associated spaces will be of importance.

Definition 1. Let

$$
\psi_{t}:=\operatorname{diag}\left(A_{t} X_{t-}\right)-A_{t} \operatorname{diag}\left(X_{t-}\right)-\operatorname{diag}\left(X_{t-}\right) A_{t}^{\top} .
$$

Then $\psi$ is a predictable process taking values in the symmetric, positive semidefinite matrices in $\mathbb{R}^{N \times N}$, with the property that

$$
\mathbb{E}\left[\left(\int_{] 0, t]} Z_{u}^{\top} \mathrm{d} M_{u}\right)^{2}\right]=\int_{] 0, t]} \mathbb{E}\left[Z_{u}^{\top} \psi_{u} Z_{u}\right] \mathrm{d} u
$$

for any $t$ and any predictable processes $Z$ of correct dimension (see [4]). For simplicity, we write

$$
\|z\|_{M_{t}}^{2}:=z^{\top} \psi_{t} z
$$

and note that this is a stochastic seminorm.

We define the following spaces of processes:

- $Y \in S^{2}$ if $\mathbb{E}\left[\sup _{t \in \mathbb{R}^{+}} Y_{t}^{2}\right]<\infty$ and $Y$ is càdlàg,

- $Z \in H_{M}^{2}$ if $\mathbb{E}\left[\int_{] 0, \infty]}\left\|Z_{t}\right\|_{M_{t}}^{2} \mathrm{~d} t\right]<\infty$ and $Z$ is predictable,

- $Z \sim_{M} Z^{\prime}$ if $\left\|Z_{t}-Z_{t}^{\prime}\right\|_{M_{t}}=0$ for almost all $t$,

- $Y \in S_{t \text {-loc }}^{2}$ if $\left\{Y_{t} I_{t \leq T}\right\} \in S^{2}$ for any $T<\infty$, and similarly $H_{M, t \text {-loc }}^{2}$.

We note that the definition of $S_{t \text {-loc }}^{2}$ is not the same as the set of processes locally in $S^{2}$ as usually understood in stochastic analysis, as the requirement is for any deterministic $T$, rather than for a sequence of stopping times.

The basic existence theorem for BSDEs in this setting is the following.

Theorem 1. Let $T$ be a finite deterministic time, and $f: \Omega \times[0, T] \times \mathbb{R} \times \mathbb{R}^{N} \rightarrow \mathbb{R}$ be a predictable function. If $f$ is uniformly Lipschitz in $y$ and $z$, that is, there exists a constant $c>0$ such that

$$
\left|f(\omega, t, y, z)-f\left(\omega, t, y^{\prime}, z^{\prime}\right)\right|^{2} \leq c\left(\left|y-y^{\prime}\right|^{2}+\left\|z-z^{\prime}\right\|_{M_{t}}^{2}\right)
$$


and

$$
\mathbb{E}\left[\int_{] 0, T]}|f(\omega, t, 0,0)|^{2} \mathrm{~d} t\right]<\infty,
$$

then, for any $\xi \in L^{2}\left(\mathcal{F}_{T}\right)$, there exists a unique solution $(Y, Z) \in S^{2} \times H_{M}^{2}$ to the BSDE

$$
\xi=Y_{t}-\int_{] t, T]} f\left(\omega, u, Y_{u-}, Z_{u}\right) \mathrm{d} u+\int_{] t, T]} Z_{u}^{\top} \mathrm{d} M_{u} .
$$

Proof. For the finite state case, this result is given in [4]. For the infinite state case, we use the martingale representation result established in [4], which naturally extends to general spaces, coupled with the existence result for BSDEs in general spaces established in [6].

We recall a key definition from [7].

Definition 2. Consider $A$ and $B$ (possibly infinite) rate matrices, that is, matrices with $A_{i j} \geq 0$ for $i \neq j$ and $\sum_{i} A_{i j}=0$ for all $j$, and similarly for $B$. We write $\mathbb{E}^{A}$ for the expectation under the measure where $X$ is a Markov chain with rate matrix $A$.

For $\gamma>0$, we shall say that $B$ is $\gamma$-controlled by $A$ whenever $B-\gamma A$ is also a rate matrix, and the diagonal entries of $B-\gamma A$ are at most $-\gamma$. If $B$ is $\gamma$-controlled by $A$, then we shall write $A \preceq_{\gamma} B$.

If $A \preceq_{\gamma} B$ and $B \preceq_{\gamma} A$, we shall write $A \sim_{\gamma} B$.

A key result in the analysis of BSDEs is the comparison theorem. In the case of BSDEs with Markov chain noise, and in general for BSDEs with jumps, a further condition is required to ensure that the result holds. In [3] and [6], a general condition under which the comparison theorem holds was presented, and in [5] a condition specific to finite state Markov chain BSDEs was also given. We here give another variant, which in some sense underlies the others.

Definition 3. For a driver $f$, we say that $f$ is $\gamma$-balanced if there exists a random field $\lambda: \Omega \times \mathbb{R}^{+} \times \mathbb{R}^{N} \times \mathbb{R}^{N} \rightarrow \mathbb{R}^{N}$, with $\lambda\left(\cdot, \cdot, z, z^{\prime}\right)$ predictable and $\lambda(\omega, t, \cdot, \cdot)$ Borel measurable, such that

- $f(\omega, t, y, z)-f\left(\omega, t, y, z^{\prime}\right)=\left(z-z^{\prime}\right)^{\top}\left(\lambda\left(\omega, t, z, z^{\prime}\right)-A X_{t-}\right)$,

- for each $e_{i} \in \mathcal{X}$,

$$
\frac{e_{i}^{\top} \lambda\left(\omega, t, z, z^{\prime}\right)}{e_{i}^{\top} A X_{t-}} \in\left[\gamma, \gamma^{-1}\right]
$$

for some $\gamma>0$, where $0 / 0:=1$,

- $\mathbf{1}^{\top} \lambda\left(\omega, t, z, z^{\prime}\right) \equiv 0$, for $\mathbf{1} \in \mathbb{R}^{N}$ (the vector with all entries 1 ), and

- $\lambda\left(\omega, t, z+\alpha \mathbf{1}, z^{\prime}\right)=\lambda\left(\omega, t, z, z^{\prime}\right)$ for all $\alpha \in \mathbb{R}$.

For simplicity, we write $\lambda_{t}^{z, z^{\prime}}$ for $\lambda\left(\omega, t, z, z^{\prime}\right)$.

Remark 2. A minor variation on the proof of [7, Lemma 12] shows that for $f$ to be $\gamma$-balanced it is sufficient, but not necessary, that, for all $y, z$, and $z^{\prime}$,

$$
\frac{f(\omega, t, y, z)-f\left(\omega, t, y, z^{\prime}\right)}{\left\|z-z^{\prime}\right\|_{M_{t}}^{2}}\left(z-z^{\prime}\right)^{\top} \Delta M_{t}>-1+\gamma
$$


up to indistinguishability. A significant case where this condition may not hold is when $f(\omega, t, y, z)=z^{\top}(B-A) X_{t-}$, for some $B \sim_{\gamma} A$. Here we see directly that $f$ is $\gamma$-balanced with $\lambda_{t}^{z, z^{\prime}}=B X_{t-}$.

Lemma 1. If $f$ is $\gamma$-balanced, then it is Lipschitz with respect to $z$ under the $\|\cdot\|_{M_{t}}$ seminorm.

Proof. For each $v \in \mathbb{R}^{N}$, we can write

$$
\|v\|_{M_{t}}^{2}=v^{\top} \psi_{t} v=\sum_{e_{i} \neq X_{t-}}\left(e_{i}^{\top} v_{i}-X_{t-}^{\top} v\right)^{2}\left(e_{i}^{\top} A X_{t-}\right) .
$$

Therefore,

$$
\begin{aligned}
\left(v^{\top} A X_{t-}\right)^{2} & =\sum_{\left\{i, j: e_{i}, e_{j} \neq X_{t-}\right\}}\left(e_{i}^{\top} v_{i}-X_{t-}^{\top} v\right)\left(e_{j}^{\top} v_{i}-X_{t-}^{\top} v\right)\left(e_{i}^{\top} A X_{t-}\right)\left(e_{j}^{\top} A X_{t-}\right) \\
& \leq \sum_{\left\{i: e_{i} \neq X_{t-}\right\}}\left(e_{i}^{\top} v_{i}-X_{t-}^{\top} v\right)^{2}\left(e_{i}^{\top} A X_{t-}\right) \sum_{\left\{j: e_{j} \neq X_{t-}\right\}}\left(e_{j}^{\top} A X_{t-}\right) \\
& =\left|X_{t-} A X_{t-}\right|\|v\|_{M}^{2} .
\end{aligned}
$$

As we assumed, $\left|X_{t-} A X_{t-}\right|$ is bounded; this shows that $v \mapsto v^{\top} A X_{t-}$ is Lipschitz in the $\|\cdot\|_{M_{t}}$ seminorm. By assumption, we have

$$
f(\omega, t, y, z)-f\left(\omega, t, y, z^{\prime}\right)=\left(z-z^{\prime}\right)^{\top}\left(\lambda_{t}^{z, z^{\prime}}-A X_{t-}\right),
$$

so, as $\lambda_{t}^{z, z^{\prime}}=D A X_{t-}$, for $D$ some diagonal matrix with diagonal entries in $\left[\gamma, \gamma^{-1}\right]$, we know

$$
f(\omega, t, y, z)-f\left(\omega, t, y, z^{\prime}\right)=\left(z-z^{\prime}\right)^{\top}(D-I)\left(A X_{t-}\right)=\left((D-I)\left(z-z^{\prime}\right)\right)^{\top}\left(A X_{t-}\right) .
$$

Therefore, by (2),

$$
\left(f(\omega, t, y, z)-f\left(\omega, t, y, z^{\prime}\right)\right)^{2} \leq \mid X_{t-} A X_{t-\mid}\left\|(D-I)\left(z-z^{\prime}\right)\right\|_{M_{t}}^{2} .
$$

As $f$ is $\gamma$-balanced we know that $f(\omega, t, y, z)=f(\omega, t, y, z+\alpha \mathbf{1})$ for any $\alpha$, so without loss of generality we can write $z-z^{\prime}$ in a form such that $X_{t-}^{\top}\left(z-z^{\prime}\right) \equiv 0$. For each $e_{i}$ we have $(D-I) e_{i}=\kappa_{i} e_{i}$ for some $\kappa_{i} \in\left[\gamma-1, \gamma^{-1}-1\right]$. As $\gamma<1$, for these $z-z^{\prime}=: \sum_{\left\{i: e_{i} \neq X_{t-}\right\}} v_{i} e_{i}$, we obtain

$$
\begin{aligned}
\left\|(D-I)\left(z-z^{\prime}\right)\right\|_{M_{t}}^{2} & =\left\|\sum_{\left\{i: e_{i} \neq X_{t-}\right\}} \kappa_{i} v_{i} e_{i}\right\|_{M_{t}}^{2} \\
& =\sum_{\left\{i: e_{i} \neq X_{t-}\right\}}\left(\kappa_{i} v_{i}\right)^{2}\left(e_{i}^{\top} A X_{t-}\right) \\
& \leq \gamma^{-1} \sum_{\left\{i: e_{i} \neq X_{t-}\right\}} v_{i}^{2}\left(e_{i}^{\top} A X_{t-}\right) \\
& =\gamma^{-1}\left\|z-z^{\prime}\right\|_{M_{t}}^{2},
\end{aligned}
$$

which yields the result.

In the following lemma, we use the notion of the essential supremum of a family of random variables (as opposed to the essential supremum of a single random variable). This is similar conceptually to the supremum taken pointwise for each $\omega$, with more care taken to ensure measurability and to prevent sets of measure zero contaminating the result. A construction and discussion of this concept can be found in [15, Appendix A5]. 
Lemma 2. Let $\{f(u ; \omega, t, y, z)\}_{u \in U}$ be a family of $\gamma$-balanced drivers. Consider the function

$$
g(\omega, t, y, z):=\underset{u \in U}{\operatorname{ess} \sup }\{f(u ; \omega, t, y, z)\},
$$

and suppose that $g(\omega, t, y, z)$ is almost surely (a.s.) finite for all $(t, y, z)$. Then $g$ is also $\gamma$-balanced, and similarly for $\operatorname{ess~} \inf _{u \in U} f$.

Proof. Suppose first that for almost all $\omega$, and for all $t, y$, and $z$, the supremum is attained. Then, omitting $\omega, t$, and $y$ for notational simplicity, there exists some predictable control $u^{*}$ such that

$$
g(z)-g(\bar{z}) \leq f\left(u^{*} ; z\right)-f\left(u^{*} ; \bar{z}\right)=(z-\bar{z})\left(\lambda^{*}-A X_{t-}\right)
$$

for some $\lambda^{*}$ satisfying the requirements of Definition 3 for $f\left(u^{*} ; \cdot\right)$. Similarly, there exists some $u_{*}, \lambda_{*}$ such that

$$
g(z)-g(\bar{z}) \geq f\left(u_{*} ; z\right)-f\left(u_{*} ; \bar{z}\right)=(z-\bar{z})\left(\lambda_{*}-A X_{t-}\right) .
$$

As $g(z)-g(\bar{z})$ is scalar, we see that, for

$$
\alpha:=\frac{g(z)-g(\bar{z})-f\left(u_{*} ; z\right)+f\left(u_{*} ; \bar{z}\right)}{f\left(u^{*} ; z\right)-f\left(u^{*} ; \bar{z}\right)-f\left(u_{*} ; z\right)+f\left(u_{*} ; \bar{z}\right)},
$$

we have

$$
g(z)-g(\bar{z})=(z-\bar{z})\left(\alpha \lambda^{*}+(1-\alpha) \lambda_{*}-A X_{t-}\right) .
$$

Finally, we note that $\lambda_{t}^{z, \bar{z}}:=\alpha \lambda^{*}+(1-\alpha) \lambda_{*}$ satisfies all the requirements of Definition 3 .

If the supremum is not attainable, then it is possible (but tedious) to construct an appropriate approximation sequence and to show that the corresponding vectors $\lambda$ have a convergent subsequence in $\ell_{2}$, using the boundedness properties of $\lambda$. The limit of this sequence will then satisfy the requirements of the Lemma. The details are left to the reader.

Theorem 2. (Finite-time comparison theorem.) Let $(Y, Z)$ and $\left(Y^{\prime}, Z^{\prime}\right)$ be the solutions to two BSDEs with drivers $f$ and $f^{\prime}$. Suppose that $f$ is $\gamma$-balanced and $f(\omega, t, y, z) \geq f^{\prime}(\omega, t, y, z)$ for all $(y, z), \mathrm{d} t \times \mathrm{d} \mathbb{P}$-a.s. Then $Y_{T} \geq Y_{T}^{\prime}$ a.s. implies $Y_{t} \geq Y_{t}^{\prime}$ a.s. up to indistinguishability.

The finite-time comparison theorem is easy to deduce (see, for example, [9]) from the following lemma.

Lemma 3. If $f$ is $\gamma$-balanced then, for any predictable processes $Z, Z^{\prime} \in H_{M, t-\mathrm{loc}}^{2}$, any process $Y \in S_{t-\mathrm{loc}}^{2}$, and any $T<\infty$, there exists a probability measure $\mathbb{Q}^{T}$ equivalent to $\mathbb{P}$ such that

$$
\tilde{M}_{t}=\int_{] 0, t \wedge T]}\left(f\left(\omega, s, Y_{s-}, Z_{s}\right)-f\left(\omega, s, Y_{s-}, Z_{s}^{\prime}\right)\right) \mathrm{d} s+\int_{] 0, t \wedge T]}\left(Z_{s}-Z_{s}^{\prime}\right)^{\top} \mathrm{d} M_{s}
$$

is a $\mathbb{Q}^{T}$-martingale.

Proof. Let $\mathbb{Q}^{T}$ be the measure under which $X$ jumps, at time $t \in[0, T]$, to a state $e_{i} \neq X_{t-}$ at a rate $e_{i}^{\top} \lambda_{t}^{Z_{t}, Z_{t}^{\prime}}$, where $\lambda(\cdot)$ is the random field associated with $f$ by Definition 3. This is a predictable bounded process (as $A$ is bounded), and so the measure $\mathbb{Q}^{T}$ is well defined. Under this measure, $X$ has a semimartingale decomposition

$$
X_{t}=X_{0}+\int_{] 0, t]} \lambda_{u}^{Z_{u}, Z_{u}^{\prime}} \mathrm{d} u+\hat{M}_{t}
$$


for $\hat{M}_{t}$ a $\mathbb{Q}^{T}$ martingale. We can then verify that $\tilde{M}_{t}=\int_{] 0, t]}\left(Z_{u}-Z_{u-}\right)^{\top} d \hat{M}_{u}$, and a calculation of the predictable $\mathbb{Q}^{T}$-quadratic variation of $\tilde{M}_{t}$ guarantees the desired martingale property. As the relative rates of $X$ 's jumps under $\mathbb{P}$ and $\mathbb{Q}$ are bounded, by assumption on $\lambda$, we can deduce that $\mathbb{Q}^{T}$ and $\mathbb{P}$ are indeed equivalent measures.

The following lemma extends this measure to all time horizons, and expresses its properties in a useful manner for the study of BSDEs.

Lemma 4. Let $Y$ and $\bar{Y}$ be two processes in $S_{t-\operatorname{loc}}^{2}$ with dynamics

$$
\mathrm{d} Y_{t}=-f\left(\omega, t, Y_{t-}, Z_{t}\right) \mathrm{d} t+Z_{t}^{\top} \mathrm{d} M_{t}, \quad \mathrm{~d} \bar{Y}_{t}=-\bar{f}\left(\omega, t, \bar{Y}_{t-}, \bar{Z}_{t}\right) \mathrm{d} t+\bar{Z}_{t}^{\top} \mathrm{d} M_{t},
$$

for some processes $Z, \bar{Z} \in H_{M, t \text {-loc }}^{2}$ and some $\gamma$-balanced drivers $f, \bar{f}$. Then there exists a measure $\mathbb{Q}$ such that

$$
Y_{t}-\bar{Y}_{t}+\int_{] 0, t]}\left(f\left(\omega, s, Y_{s-}, Z_{s}\right)-\bar{f}\left(\omega, s, \bar{Y}_{s-}, Z_{s}\right)\right) \mathrm{d} s
$$

is a $\mathbb{Q}$-martingale.

Proof. For each $T$, let $\mathbb{Q}^{T}$ be the measure given by Lemma 3. Then, for $t<T$, a simple rearrangement shows that

$$
\begin{aligned}
Y_{t}- & \bar{Y}_{t}+\int_{] 0, t]}\left(f\left(\omega, s, Y_{s-}, Z_{s}\right)-\bar{f}\left(\omega, s, \bar{Y}_{s-}, Z_{s}\right)\right) \mathrm{d} s \\
& =Y_{0}-\bar{Y}_{0}+\int_{\mathrm{j} 0, t]}\left(f\left(\omega, s, Y_{s-}, Z_{s}\right)-f\left(\omega, s, Y_{s-}, \bar{Z}_{s}\right)\right) \mathrm{d} s+\int_{] 0, t]}\left(Z_{s}-\bar{Z}_{s}\right)^{\top} \mathrm{d} M_{s}
\end{aligned}
$$

by Lemma 3, this is a $\mathbb{Q}^{T}$-martingale up to time $T$. We now note that the measures $\mathbb{Q}^{T}$ are consistent, in that $\left.\mathbb{Q}^{T}\right|_{\mathcal{F}_{t}}=\left.\mathbb{Q}^{t}\right|_{\mathcal{F}_{t}}$ for any $t \leq T$. As our underlying space is the space of paths of a countable state Markov chain, which can be embedded in the space of paths in $\mathbb{R}$, Kolmogorov's extension theorem implies the existence of a measure $\mathbb{Q}$ with $\left.\mathbb{Q}\right|_{\mathcal{F}_{T}}=\left.\mathbb{Q}^{T}\right|_{\mathcal{F}_{T}}$ for all $T$.

Definition 4. Let $\mathcal{Q}_{\gamma}$ denote the family of all measures $\mathbb{Q}$ where $X$ has compensator $\lambda(\omega, t)$, for $\lambda$ a predictable process with $\mathbf{1}^{\top} \lambda \equiv 0$ and $\left(e_{i}^{\top} \lambda(\omega, t)\right) /\left(e_{i}^{\top} A X_{t-}\right) \in\left[\gamma, \gamma^{-1}\right]$ for all $i$, where $0 / 0:=1$.

Remark 3. Note that $\mathbb{P} \in \mathcal{Q}_{\gamma}$, the measures which appear in Lemma 4 are in $\mathcal{Q}_{\gamma}$, and, for any $\mathbb{Q}, \mathbb{Q}^{\prime} \in \mathcal{Q}_{\gamma}$ and any stopping time $\tau$, the measure defined by $\tilde{\mathbb{Q}}(A)=\mathbb{E}^{\mathbb{Q}}\left[\mathbb{E}^{\mathbb{Q}}\left[I_{A} \mid \mathcal{F}_{\tau}\right]\right]$ is also in $\mathbb{Q}_{\gamma}$.

It is worth noting that, in general, the measures in $\mathbb{Q}_{\gamma}$ will be singular, even though their restrictions to $\mathcal{F}_{T}$ are absolutely continuous for every $T$. The following lemma gives us a slightly stronger result.

Lemma 5. Let $\tau$ be a stopping time with $\tau<\infty$ a.s., and let $\mathbb{Q} \in \mathcal{Q}_{\gamma}$. Then $\left.\mathbb{Q}\right|_{\mathcal{F}_{\tau}}$ and $\left.\mathbb{P}\right|_{\mathcal{F}_{\tau}}$ are equivalent.

Proof. As the relative rates of jumping are bounded, we know that $\left.\mathbb{P}\right|_{\mathcal{F}_{T}}$ and $\left.\mathbb{Q}\right|_{\mathcal{F}_{T}}$ are equivalent for any $T<\infty$. Let $A \in \mathcal{F}_{\tau}$. Then, by the definition of $\mathcal{F}_{\tau}$,

$$
\mathbb{P}(A \cap\{\tau<T\})=0 \Longleftrightarrow \mathbb{Q}(A \cap\{\tau<T\})=0 .
$$


Now, as $\tau$ is a.s. finite valued, by the monotone convergence theorem

$$
\lim _{T \rightarrow \infty} \mathbb{P}(A \cap\{\tau<T\})=\mathbb{P}(A)
$$

and similarly for $\mathbb{Q}$. Hence,

$$
\begin{aligned}
\mathbb{P}(A)=0 & \Longleftrightarrow \mathbb{P}(A \cap\{\tau<T\})=0 \quad \text { for all } T \\
& \Longleftrightarrow \mathbb{Q}(A \cap\{\tau<T\})=0 \quad \text { for all } T \\
& \Longleftrightarrow \mathbb{Q}(A)=0,
\end{aligned}
$$

and so $\mathbb{P}$ and $\mathbb{Q}$ are equivalent on $\mathcal{F}_{\tau}$.

\section{BSDEs to Stopping times}

In this section, we make great use of the following version of Markov's inequality: 'for any stopping time $\tau$ and any $T>0, \mathbb{E}\left[I_{\tau>T} \mid \mathcal{F}_{t}\right] \leq \mathbb{E}\left[\tau^{1+\beta} \mid \mathcal{F}_{t}\right] T^{-(1+\beta)}$.

Our key result is as follows.

Theorem 3. Suppose that $\xi$ is $\mathcal{F}_{\tau}$-measurable and that, for some nondecreasing functions $K, \tilde{K}: \mathbb{R}^{+} \rightarrow[1, \infty[$ and some constants $\beta, \tilde{\beta}>0$, we have

$$
\mathbb{E}^{\mathbb{Q}}\left[|\xi| \mid \mathcal{F}_{t}\right] \leq K(t), \quad \mathbb{E}^{\mathbb{Q}}\left[(1+\tau)^{1+\beta} \mid \mathcal{F}_{t}\right] \leq K(t), \quad \mathbb{E}^{\mathbb{Q}}\left[K(\tau)^{1+\tilde{\beta}} \mid \mathcal{F}_{t}\right] \leq \tilde{K}(t)
$$

all $\mathbb{P}$-a.s. for all $\mathbb{Q} \in Q_{\gamma}$ and all $t$. Let $f: \Omega \times \mathbb{R}^{+} \times \mathbb{R} \times \mathbb{R}^{N} \rightarrow \mathbb{R}$ be $\gamma$-balanced, and such that, for any $y, y^{\prime}, z$, we have

$$
|f(\omega, t, 0,0)| \leq c\left(1+t^{\hat{\beta}}\right), \quad \frac{f(\omega, t, y, z)-f\left(\omega, t, y^{\prime}, z\right)}{y-y^{\prime}} \in[-c, 0],
$$

for some $c \in \mathbb{R}$ and some $\hat{\beta} \in[0, \beta[$. Then the BSDE (1) admits a unique adapted solution satisfying the bound

$$
\left|Y_{t}\right| \leq(1+c) K(t)
$$

Proof. We first prove a solution exists. Let $\tau_{n}:=\tau \wedge n$. Define

$$
r\left(\omega, u, y, y^{\prime}, z\right):=-\frac{f(\omega, u, y, z)-f\left(\omega, t, y^{\prime}, z\right)}{y-y^{\prime}},
$$

and notice by assumption that $r \in[0, c]$ and is predictable. Define $\left(Y^{n}, Z^{n}\right)$ to be the solution to the finite-horizon BSDE

$$
Y_{t}^{n}=\xi I_{\tau \leq n}+\int_{] t, n]} f\left(\omega, u, Y_{u}^{n}, Z_{u}^{n}\right) I_{u<\tau} \mathrm{d} u-\int_{] t, n]}\left(Z_{u}^{n}\right)^{\top} \mathrm{d} M_{u} .
$$

As $\xi$ is $\mathcal{F}_{\tau}$ measurable, we see that $Z_{u}^{n}=0$ whenever $u \geq \tau$. Now, for $n \geq m$, by Lemma 3 
and Itô's lemma, there exists a measure $\mathbb{Q}$ (depending on $n, m)$ such that

$$
\begin{aligned}
& Y_{m}^{n}=\mathbb{E}^{\mathbb{Q}}\left[\xi I_{\tau \leq n}+\int_{] \tau_{m}, \tau_{n}\right]} f\left(\omega, u, Y_{u-}^{n}, 0\right) \mathrm{d} u \mid \mathcal{F}_{m}\right] \\
&=\mathbb{E}^{\mathbb{Q}}\left[\exp \left(-\int_{] \tau_{m}, \tau_{n}\right]} r\left(\omega, u, Y_{u-}^{n}, 0,0\right) \mathrm{d} u\right)\right. \\
&\left.\times\left(\xi I_{\tau \leq n}+\int_{] \tau_{m}, \tau_{n}\right]} f(\omega, u, 0,0) \mathrm{d} u\right) \mid \mathcal{F}_{m}\right], \\
& Y_{t}^{n}-Y_{t}^{m}=\mathbb{E}^{\mathbb{Q}}\left[\left(Y_{m}^{n}-\xi I_{\tau \leq m}\right)+\int_{] t, \tau_{n}\right]} f\left(\omega, u, Y_{u-}^{n}, Z_{u}^{n}\right)-f\left(\omega, u, Y_{u-}^{m}, Z_{u}^{n}\right) \mathrm{d} u \mid \mathcal{F}_{t}\right] \\
&=\mathbb{E}^{\mathbb{Q}}\left[\exp \left(-\int_{] t, \tau_{n}\right]} r\left(\omega, u, Y_{u-}^{n}, Y_{u-}^{m}, Z_{u}^{n}\right) \mathrm{d} u\right)\left(Y_{m}^{n}-\xi I_{\tau \leq m}\right) \mid \mathcal{F}_{t}\right] .
\end{aligned}
$$

Combining these, as $r \geq 0$ and by our assumptions $|\xi| \leq K(\tau)$, we see

$$
\begin{aligned}
\left|Y_{t}^{n}-Y_{t}^{m}\right| \leq & \mathbb{E}^{\mathbb{Q}}\left[\left|\xi I_{\tau \leq n}-\xi I_{\tau \leq m}\right|+\int_{] \tau_{m}, \tau_{n}\right]}|f(\omega, u, 0,0)| \mathrm{d} u \mid \mathcal{F}_{t}\right] \\
\leq & \mathbb{E}^{\mathbb{Q}}\left[(K(\tau)) I_{\tau>m}+c\left(1+\tau^{\hat{\beta}}\right)\left(\tau_{n}-\tau_{m}\right) \mid \mathcal{F}_{t}\right] \\
\leq & \mathbb{E}^{\mathbb{Q}}\left[K(\tau) I_{\tau>m} \mid \mathcal{F}_{t}\right]+c \mathbb{E}^{\mathbb{Q}}\left[\tau\left(1+\tau^{\hat{\beta}}\right) I_{\tau>m} \mid \mathcal{F}_{t}\right] \\
\leq & \mathbb{E}^{\mathbb{Q}}\left[K(\tau)^{1+\tilde{\beta}} \mid \mathcal{F}_{t}\right]^{1 /(1+\tilde{\beta})} \mathbb{E}^{\mathbb{Q}}\left[I_{\tau>m} \mid \mathcal{F}_{t}\right]^{\tilde{\beta} /(1+\tilde{\beta})} \\
& +c \mathbb{E}^{\mathbb{Q}}\left[\tau\left(1+\tau^{\hat{\beta}}\right) \mid \mathcal{F}_{t}\right]^{1 /(1+\alpha)} \mathbb{E}^{\mathbb{Q}}\left[I_{\tau>m} \mid \mathcal{F}_{t}\right]^{\alpha /(1+\alpha)} \\
\leq & \tilde{K}(t)^{1 /(1+\tilde{\beta})}\left(\frac{K(t)}{m^{1+\beta}}\right)^{\tilde{\beta} /(1+\tilde{\beta})}+2 c K(t)^{1 / \alpha}\left(\frac{K(t)}{m^{1+\beta}}\right)^{\alpha /(1+\alpha)},
\end{aligned}
$$

where $\alpha:=(\beta-\hat{\beta}) /(1+\hat{\beta})$. Hence, writing $\tilde{\alpha}:=(1+\beta)(\tilde{\beta} /(1+\tilde{\beta}) \wedge \alpha /(1+\alpha))$, as we know that $\tilde{\alpha}>0$, we have a bound of the form

$$
\left|Y_{t}^{n}-Y_{t}^{m}\right| \leq \hat{K}(t) m^{-\tilde{\alpha}},
$$

for some nondecreasing function $\hat{K}(t)$, and so $Y^{n}$ is a Cauchy sequence (which converges a.s. in $\omega$, uniformly on compacts in time, and hence uniformly on compacts in probability).

Now, define $Y_{t}:=\lim _{n} Y_{t}^{n}$. It is easy to see that this satisfies the desired dynamics. Furthermore, for some measure $\mathbb{Q}$ we have the representation

$$
Y_{t}=\mathbb{E}^{\mathbb{Q}}\left[\exp \left(-\int_{] t, \tau]} r\left(\omega, u, Y_{u-}, 0,0\right) \mathrm{d} u\right)\left(\xi+\int_{] t, \tau]} f(\omega, u, 0,0) \mathrm{d} u\right) \mid \mathcal{F}_{t}\right],
$$

from which, given our assumptions on $f$ and $\tau$, we can see that

$$
\left|Y_{t}\right| \leq(1+c) K(t)
$$

Now, suppose that we have two solutions $Y$ and $\tilde{Y}$. Then, from the assumed bound and the fact that $Y_{T}=\tilde{Y}_{T}=\xi$ on the set $\tau \leq T$, we can see that, for some measure $\mathbb{Q}$ given by 
Lemma 3,

$$
\begin{aligned}
\mathbb{E}^{\mathbb{P}}\left[\left|Y_{t}-\tilde{Y}_{t}\right|\right] & =\mathbb{E}^{\mathbb{P}}\left[\left|\mathbb{E}^{\mathbb{Q}}\left[\exp \left(-\int_{] t, T \wedge \tau]} r\left(\omega, u, Y_{u-}, \tilde{Y}_{u-}, Z_{t}\right) \mathrm{d} u\right)\left(Y_{T}-\tilde{Y}_{T}\right) \mid \tilde{F}_{t}\right]\right|\right] \\
& \leq \mathbb{E}^{\mathbb{P}}\left[\mathbb{E}^{\mathbb{Q}}\left[\left|Y_{T}-\tilde{Y}_{T}\right| \mid \mathcal{F}_{t}\right]\right] \\
& \leq \mathbb{E}^{\mathbb{P}}\left[2(1+c) \mathbb{E}^{\mathbb{Q}}\left[K(T) I_{\tau>T} \mid \mathcal{F}_{t}\right]\right] \\
& \leq 2(1+c) \mathbb{E}^{\mathbb{P}}\left[\mathbb{E}^{\mathbb{Q}}\left[K(\tau) I_{\tau>T} \mid \mathcal{F}_{t}\right]\right] \\
& \leq 2(1+c) \mathbb{E}^{\mathbb{P}}\left[\mathbb{E}^{\mathbb{Q}}\left[K(\tau)^{1+\beta} \mid \mathcal{F}_{t}\right]\right]^{1 /(1+\beta)} \mathbb{E}^{\mathbb{P}}\left[\mathbb{E}^{\mathbb{Q}}\left[I_{\tau>T} \mid \mathcal{F}_{t}\right]\right]^{\beta /(1+\beta)} \\
& \leq 2(1+c) \tilde{K}(0)^{1 /(1+\beta)}\left(\frac{K(t)}{T^{1+\beta}}\right)^{\beta /(1+\beta)} \\
& \leq 2(1+c) \tilde{K}(0)^{1 /(1+\beta)} K(t)^{\beta /(1+\beta)} T^{-\beta}
\end{aligned}
$$

As $\beta>0$, letting $T \rightarrow \infty$ implies that $\mathbb{E}^{\mathbb{P}}\left[\left|Y_{t}-\tilde{Y}_{t}\right|\right]=0$ for all $t$, and so the solutions coincide a.s. As the solutions are càdlàg, they agree up to indistinguishability.

Remark 4. We emphasise that the above proof permits the case when $f$ does not depend on $y$. In fact, the requirement that $f$ is monotone decreasing is not strictly necessary for our proof. It is enough to guarantee that the discounting terms given by the integrals $\int_{] s, t]} r\left(\omega, u, y, y^{\prime}, z\right) \mathrm{d} u$ are uniformly bounded above for all $s, t$ and all processes $y, y^{\prime}, z$, as this just introduces a positive constant into our estimates, which will then also appear in the bound on $Y_{t}$.

We now extend our existence result to drivers which are only 'locally' $\gamma$-balanced and Lipschitz (in $y$ ), under slightly more restrictive assumptions on the terminal condition and the driver evaluated at zero.

Theorem 4. Suppose that $\xi$ is $\mathcal{F}_{\tau}$-measurable and for some constant $k$ we have

$$
|\xi| \leq k, \quad|f(\omega, t, 0,0)| \leq k, \quad \mathbb{E}^{\mathbb{Q}}\left[(\tau-t)^{+} \mid \mathscr{F}_{t}\right] \leq k
$$

and, for some $\beta>0$ and some nondecreasing functions $K, \tilde{K}: \mathbb{R} \rightarrow[1, \infty[$, we have

$$
\mathbb{E}^{\mathbb{Q}}\left[(1+\tau)^{1+\beta} \mid \mathcal{F}_{t}\right] \leq K(t), \quad \mathbb{E}^{\mathbb{Q}}\left[K(\tau)^{1+\beta} \mid \mathcal{F}_{t}\right] \leq \tilde{K}(t),
$$

all $\mathbb{P}$-a.s. for all $\mathbb{Q} \in \mathcal{Q}_{\gamma}$ and all $t$.

For $y, n \in \mathbb{R}$, let $y^{(n)}:=(-n) \vee y \wedge n$, and for $z \in \mathbb{R}^{N}$ let $z^{(n)}$ be the vector with components $\left(e_{i}^{\top} z\right)^{(n)}$. For $f: \Omega \times \mathbb{R}^{+} \times \mathbb{R} \times \mathbb{R}^{N} \rightarrow \mathbb{R}$ a predictable function, suppose that

$$
f^{(n)}(\omega, t, y, z):=f\left(\omega, t, y^{(n)},\left(z-\left(X_{t-}^{\top} z\right) \mathbf{1}\right)^{(n)}\right)
$$

satisfies the requirements of Theorem 3 for every $n>0$. (That is, $f^{(n)}$ is $\gamma$-balanced in $z$ and Lipschitz decreasing in $y$, where $\gamma$ and the Lipschitz constant can depend on $n$.) Then the $B S D E(1)$ with driver $f$ admits a unique bounded solution.

Proof. First note that, as $f^{(n)}$ satisfies the requirements of Theorem 3 for any $n$, the BSDE with driver $f^{(n)}$ admits a unique solution $\left(Y^{n}, Z^{n}\right)$. We know that this solution has a representation

$$
Y_{t}^{n}=\mathbb{E}^{\mathbb{Q}}\left[\exp \left(-\int_{] t, \tau]} r\left(\omega, u, Y_{u-}^{n}, 0,0\right) \mathrm{d} u\right)\left(\xi+\int_{] t, \tau]} f^{(n)}(\omega, u, 0,0) \mathrm{d} u\right) \mid \mathcal{F}_{t}\right]
$$


for some $\mathbb{Q} \in \mathcal{Q}_{\gamma^{n}}$, where $r$ is as in (3), and so

$$
\left|Y_{t}^{n}\right| \leq \mathbb{E}^{\mathbb{Q}}\left[|\xi|+\int_{] t, \tau]}\left|f^{(n)}(\omega, u, 0,0)\right| \mathrm{d} u \mid \mathcal{F}_{t}\right] \leq k+k \mathbb{E}^{\mathbb{Q}}\left[(\tau-t)^{+} \mid \mathscr{F}_{t}\right] \leq k(1+k) .
$$

From the fact that $\Delta Y_{t}^{n}=\left(Z_{t}^{n}\right)^{\top} \Delta X_{t}$ and $\Delta X_{t}=e_{i}-e_{j}$ for some $i, j$, and that jumps of $X$ are totally inaccessible and $Z$ is predictable, we see that $\left|e_{i}^{\top} Z_{t}^{n}\right| \leq 2 k(1+k)$ up to equivalence $\sim_{M}$. Hence, we have a bound on $Y^{n}, Z^{n}$ independent of $n$. Therefore, provided that $n \geq 2 k(1+k)$, we see that $Y_{t}^{n}, Z_{t}^{n}$ is a solution for the $\operatorname{BSDE}$ (1) with driver $f$, as the truncation will have no effect.

Conversely, suppose that we had two bounded solutions $(Y, Z),\left(Y^{\prime}, Z^{\prime}\right)$. Then we could set $n>k^{\prime}$, where $k^{\prime}$ is a bound on the solutions, so that the truncation has no effect, and both processes would solve the BSDE with driver $f^{(n)}$. However, this BSDE satisfies Theorem 3 and so admits unique solutions; hence, we have a contradiction.

Remark 5. The peculiar definition of $f^{(n)}$ in Theorem 4 (in particular, the unnatural use of $\left(z-\left(X_{t-}^{\top} z\right) \mathbf{1}\right)^{(n)}$ rather than $\left.z^{(n)}\right)$, is simply to ensure that $f^{(n)}$ is Lipschitz continuous in the $\|\cdot\|_{M_{t}}$ seminorm whenever $f$ is, as the basic truncation $z \mapsto z^{(n)}$ is not invariant under $\|\cdot\|_{M_{t}}$ equivalence.

The conditions of these statements may seem unusual and restrictive; however, the following lemma gives a key example when they are satisfied. It is an immediate consequence of Corollary 1 , below, and so is stated without proof.

Lemma 6. Let $\tau$ be the first hitting time of a set $\Xi \subseteq X$. Let $\xi$ be a random variable of the form

$$
\xi=g\left(\tau, X_{\tau}\right),
$$

for some function $g$ with $g(t, x) \leq k\left(1+t^{\beta}\right)$ for some $k, \beta>0$. Then there exist functions $K, \tilde{K}$ satisfying the requirements of Theorem 3 .

Remark 6. Theorem 3 (but not Theorem 4) would work equally well for Brownian-motionbased BSDEs, with the corresponding definition of the family of measures $\mathcal{Q}_{\gamma}$.

Theorem 5. (Comparison theorem.) Let $(Y, Z)$ and $\left(Y^{\prime}, Z^{\prime}\right)$ be the solutions to two BSDEs with drivers $f, f^{\prime}$ and terminal values $\xi$, $\xi^{\prime}$ satisfying the conditions of Theorem 3 or 4 . Suppose that $f(\omega, t, y, z) \geq f^{\prime}(\omega, t, y, z)$ for all $(y, z), \mathrm{d} t \times \mathrm{d} \mathbb{P}$-a.s. Then $\xi \geq \xi^{\prime}$ a.s. implies $Y_{t} \geq Y_{t}^{\prime}$ a.s. up to indistinguishability. Furthermore, $Y_{t}=Y_{t}^{\prime}$ a.s. on $A \in \mathcal{F}_{t}$ if and only if $Y_{s}=Y_{s}^{\prime}$ a.s. on A for each $s>t$ and $f\left(\omega, s, Y_{s-}, Z_{s}\right)=f^{\prime}\left(\omega, s, Y_{s-}^{\prime}, Z_{s}^{\prime}\right)$ on $\left.\left.A \times\right] t, \tau\right], \mathrm{d} \mathbb{P} \times \mathrm{d} t$-a.s.

Proof. From Lemma 4, we know that there is a measure $\mathbb{Q}$ such that, for any stopping time $\tau^{*} \leq \tau$,

$$
Y_{t}-Y_{t}^{\prime}=\mathbb{E}^{\mathbb{Q}}\left[\left(Y_{\tau^{*}}-Y_{\tau^{*}}^{\prime}+\int_{] t, \tau^{*}\right]} f\left(\omega, u, Y_{u-}, Z_{u}\right)-f^{\prime}\left(\omega, u, Y_{u-}^{\prime}, Z_{u}\right) \mathrm{d} u\right) \mid \mathcal{F}_{t}\right] .
$$

From Lemma 5, we know that the stated assumptions, $\xi \geq \xi^{\prime}$ and $f \geq f^{\prime}$, hold both $\mathbb{P}$-a.s. and $\mathbb{Q}$-a.s. Suppose that we have a set $B \in \mathcal{F}_{t}$ such that $Y_{t}-Y_{t}^{\prime}<0$ on $B$. Then define the stopping time

$$
\tau^{*}=\inf \left\{s \geq t: Y_{s}-Y_{s}^{\prime} \geq 0\right\} \leq \tau
$$


From this and the definition of $r$ in (3), it is easy to deduce that

$$
I_{B}\left|Y_{t}-Y_{t}^{\prime}\right| \leq \mathbb{E}^{\mathbb{Q}}\left[-\int_{] t, \tau^{*}\right]} r\left(\omega, u, Y_{u-}, Y_{u-}^{\prime}, Z_{u}\right) I_{B}\left|Y_{u-}-Y_{u-}^{\prime}\right| \mathrm{d} u \mid \mathcal{F}_{t}\right] .
$$

However, as $r \geq 0$, the right-hand side is nonpositive, so $I_{B}\left|Y_{t}-Y_{t}^{\prime}\right|=0$, that is, $Y_{t} \geq Y_{t}^{\prime}$ except on a null set.

Now, for any $A \in \mathcal{F}_{t}$, it is easy to verify from the representation

$$
Y_{t}-Y_{t}^{\prime}=\mathbb{E}^{\mathbb{Q}}\left[\exp \left(-\int_{] t, \tau]} r\left(\omega, u, Y_{u-}, Y_{u-}^{\prime}, Z_{u}\right) \mathrm{d} u\right)\left(\xi-\xi^{\prime}\right) \mid \mathcal{F}_{t}\right]
$$

that $I_{A}\left(Y_{t}-Y_{t}^{\prime}\right)=0$ if and only if $I_{A}\left(Y_{s}-Y_{s}^{\prime}\right)=0$ a.s. for each $s>t$ and thence also that $f\left(\omega, s, Y_{s-}, Z_{s}\right)=f^{\prime}\left(\omega, s, Y_{s-}^{\prime}, Z_{s}^{\prime}\right)$ on $\left.\left.A \times\right] t, \tau\right], \mathrm{d} \mathbb{P} \times \mathrm{d} t$-a.s.

For completeness, we now state a useful result which gives us a solution of the form $Y_{t}=u\left(t, X_{t}\right)$. In [8, Corollary 2] this result is stated, but only for the case $N<\infty$ and $T$ deterministic.

Theorem 6. Let $\tau$ be the first hitting time of a set $\Xi \subset \mathcal{X}$, and let $Y$ be the solution to a $B S D E$ with Markovian terminal condition $Y_{\tau}=\phi\left(\tau, X_{\tau}\right)$, for some bounded deterministic function $\phi: \mathbb{R}^{+} \times \mathbb{R}^{N} \rightarrow \mathbb{R}$. Suppose that $f$ satisfies the requirements of Theorem 3 or 4 and is Markovian in the sense that $f(\omega, t, y, z)=\tilde{f}\left(X_{t-}, t, y, z\right)$ for some $\tilde{f}$. Then there exists a measurable function $u: \mathbb{R}^{+} \times \mathcal{X} \rightarrow \mathbb{R}$ such that $Y_{t}=u\left(t, X_{t}\right)$ for all $t \leq \tau$. Furthermore, $u$ satisfies

- $u(t, x)=\phi(t, x)$ on $\mathbb{R}^{+} \times \Xi$,

- the associated vector $\boldsymbol{u}_{t}$ defined by $e_{i}^{\top} \boldsymbol{u}_{t}=u\left(t, e_{i}\right)$ satisfies the $N$-dimensional ODE system

$$
\mathrm{d} \boldsymbol{u}_{t}=-\left(\boldsymbol{f}\left(t, \boldsymbol{u}_{t}\right)+A^{\top} \boldsymbol{u}_{t}\right) \mathrm{d} t, \quad \text { for } t<\tau,
$$

where $e_{i}^{\top} \boldsymbol{f}(t, \boldsymbol{u}):=\tilde{f}\left(e_{i}, t, e_{i}^{\top} \boldsymbol{u}, \boldsymbol{u}\right)$,

- and the solution process $Z$ is given by $Z_{t}=\boldsymbol{u}_{t}$, up to equivalence $\sim_{M}$; in particular, note that the BSDE has a solution $Z$ which is deterministic.

Proof. First note that, by the Markov property, we have

$$
\begin{aligned}
Y_{t} & =\mathbb{E}\left[\phi\left(\tau, X_{\tau}\right)+\int_{] t, \tau]} f\left(X_{u-}, u, Y_{u-}, Z_{u}\right) \mathrm{d} u \mid \mathcal{F}_{t}\right] \\
& =\mathbb{E}\left[\phi\left(\tau, X_{\tau}\right)+\int_{] t, \tau]} f\left(X_{u-}, u, Y_{u-}, Z_{u}\right) \mathrm{d} u \mid X_{t}\right] \\
& =u\left(t, X_{t}\right),
\end{aligned}
$$

for some function $u$, where the third line follows from the Doob-Dynkin lemma, which implies that for each $t$, as $Y_{t}$ is $\sigma\left(X_{t}\right)$-measurable, $Y_{t}$ is equal to a measurable function of $X_{t}$. Right-continuity of $Y$ in $t$ ensures that the function $u$ is measurable in the product space. Clearly, $u(t, x)=\phi(t, x)$ on the set $t=\tau$, that is, on $\mathbb{R}^{+} \times \Xi$. Applying [8, Theorem 3.2] (modified trivially to allow $N=\infty$ if necessary) we have the desired dynamics of $u$ and the statement $Z_{t}=\boldsymbol{u}_{t}$. 
We can also obtain a version of this result under which $Y$ does not depend on time.

Theorem 7. Let $\tau$ be the first hitting time of a set $\Xi \subseteq X$ and let $f: X \times \mathbb{R}^{+} \times \mathbb{R}^{N} \rightarrow \mathbb{R}$ satisfy the conditions of Theorem 4. Consider the BSDE

$$
Y_{t}=\phi\left(X_{\tau}\right)-\int_{] t, \tau]} f\left(X_{u-}, Y_{u-}, Z_{u}\right) \mathrm{d} u+\int_{] t, \tau]} Z_{u}^{\top} \mathrm{d} M_{u},
$$

where $\phi$ is a bounded function $\mathcal{X} \rightarrow \mathbb{R}$. Then there exists a bounded function $u: \mathcal{X} \rightarrow \mathbb{R}$ such that $Y_{t}=u\left(X_{t}\right)$ and $e_{i}^{\top} Z_{t}=u\left(e_{i}\right)$, so $Z$ is constant. The vector $\boldsymbol{u}$ defined by $e_{i}^{\top} \boldsymbol{u}=u\left(e_{i}\right)$ satisfies the equation

$$
f\left(x, x^{\top} \boldsymbol{u}, \boldsymbol{u}\right)=-\boldsymbol{u}^{\top} A x \quad \text { for } x \in X \backslash \Xi,
$$

with boundary values $u(x)=\phi(x)$ for $x \in \Xi$.

Proof. As $\tau$ is a hitting time of a Markov chain $X$, we know

$$
\begin{aligned}
Y_{t} & =\mathbb{E}\left[\phi\left(X_{\tau}\right)-\int_{] t, \tau]} f\left(X_{u-}, Y_{u-}, Z_{u}\right) \mathrm{d} u \mid \mathcal{F}_{t}\right] \\
& =\mathbb{E}\left[\phi\left(X_{\tau}\right)-\int_{] t, \tau]} f\left(X_{u-}, Y_{u-}, Z_{u}\right) \mathrm{d} u \mid X_{t}\right] .
\end{aligned}
$$

Defining a new Markov chain $\hat{X}_{s}=X_{s-t}$ for $s>t$, with hitting time $\hat{\tau}=\tau-t$, by the Markov property we have

$$
Y_{t}=\mathbb{E}\left[\phi\left(\hat{X}_{\hat{\tau}}\right)-\int_{] 0, \hat{\tau}]} f\left(\hat{X}_{u-}, Y_{u-}, Z_{u}\right) \mathrm{d} u \mid \hat{X}_{0}\right]
$$

and, as the right-hand side does not depend on $t$, we see that $Y_{t}$ is a function purely of $\hat{X}_{0}=X_{t}$.

Let $\hat{A}$ be the modification of the rate matrix such that $\hat{A} x=A x$ for $x \notin \Xi$ and $\hat{A} x=0$ for $x \in \Xi$. This agrees with $A$ on the set $t<\tau$, so, from Theorem 6 , we see that there exists a vector $\boldsymbol{u}$ such that $Y_{t}=X_{t-}^{\top} \boldsymbol{u}, Z_{t}=\boldsymbol{u}$, and

$$
0=-\left(\boldsymbol{f}(\boldsymbol{u})+\hat{A}^{\top} \boldsymbol{u}\right) \mathrm{d} t \text { for } t<\tau
$$

However, this equation does not depend on $t$, and so $\boldsymbol{f}(\boldsymbol{u})+\hat{A}^{\top} \boldsymbol{u}=0$. Premultiplying by $x \in X$ and using the relation between $A$ and $\hat{A}$ and the boundary conditions, we obtain the desired equation. Boundedness of $u$ is trivial from this representation, as we have assumed that $f$ and $\phi$ are bounded.

\section{Exponential hitting time bounds}

We now seek to show that, when $\tau$ is the hitting time of a state of the chain, the required $\tau$-integrability assumptions of Theorem 3 are satisfied. This is done by examining the exponential ergodicity of the Markov chain under perturbations of the rate matrix.

A key result of [7] is that, when the driver $f$ is $\gamma$-balanced, does not depend on time or $Y$, and depends on $\omega$ only through $X_{t}(\omega)$, the measures $\mathbb{Q}$ and $\mathbb{P}$ established by Lemma 4 have closely related ergodic properties. In particular, if the underlying Markov chain is uniformly ergodic under $\mathbb{P}$, then it is also uniformly ergodic under $\mathbb{Q}$, and the rate of convergence to the ergodic distribution can be uniformly bounded for every $\mathbb{Q}$ in terms of $\gamma$ and the properties of $\mathbb{P}$.

This result is of fundamental importance to our approach to BSDEs up to stopping times. Our first step is to generalise away from the Markovian assumptions on $f$, and to work only with the assumption that $f$ is $\gamma$-balanced. In this case, notions of 'ergodicity' cease to have a 
clear meaning, as the dynamics of the Markov chain under $\mathbb{Q}$ can be time-dependent. Instead, we work with a more fundamental property, that of the existence of exponential moments of the first hitting times of states of the Markov chain.

We begin with the definition of uniform ergodicity.

Definition 5. Let $\mathcal{M}$ denote the set of probability measures on $\mathcal{X}$, with the topology inherited from considering them as a convex subset of $\ell_{1}(\mathcal{X})$ (the total variation topology, with norm $\left.\|f\|_{\mathrm{TV}}=\sum_{x}|f(x)|\right)$. We write $P_{t}^{A} \mu$ for the law of $X_{t}$ given $X_{0} \sim \mu$ when $X$ evolves following the rate matrix $A$.

We say the Markov chain $X$ is uniformly ergodic if there exists a measure $\pi$ on $\mathcal{X}$, and constants $R, \rho>0$, such that

$$
\sup _{\mu \in \mathcal{M}}\left\|P_{t}^{A} \mu-\pi\right\|_{\mathrm{TV}} \leq R \mathrm{e}^{-\rho t} \quad \text { for all } t .
$$

In this case, $\pi$ is the unique invariant measure for the chain.

The following lemma is simply a variant of [18, Theorem 16.2.2(iv)] and is stated without proof, but shall be useful in our understanding of this property.

Lemma 7. Let $X$ be a uniformly ergodic Markov chain, and let $x_{C}$ be an arbitrary state. Let $\tau_{C}$ be the first hitting time of $x_{C}$. Then, for some $\beta>0$ (and hence for all $\beta$ sufficiently small), $\sup _{x \in X} \mathbb{E}\left[\mathrm{e}^{\beta \tau_{C}} \mid X_{0}=x\right]<\infty$.

We make the following basic assumption about our processes.

Assumption 1. Under the measure $\mathbb{P}$, the Markov chain $X$ has time-homogeneous rate matrix $A$ and is uniformly ergodic.

Theorem 8. Let $X, \bar{X}$ be two independent copies of the Markov chain on $\mathcal{X}$, and let these be uniformly ergodic under the measure generated by a rate matrix $A$. For some arbitrarily chosen state $\hat{x}$, let $\hat{\tau}=\inf \left\{t: X_{t}=\bar{X}_{t}=\hat{x}\right\}$, the first time $X$ and $\bar{X}$ meet in the state $\hat{x}$. Then, for any $\varepsilon>0$, there exists $\beta>0$ depending only on $\gamma$ and $A$ such that

$$
\sup _{B \succeq_{\gamma} A} \sup _{x, \bar{x}} \mathbb{E}^{B}\left[\mathrm{e}^{\beta \hat{\tau}} \mid X_{0}=x, \bar{X}_{0}=\bar{x}\right] \leq 1+\varepsilon .
$$

Proof. This is a re-expression of [7, Lemma 11, Corollary 12], where we note that the $T$ referred to here is not as stated in [7], but is rather the more restrictive stopping time used in the proof of [7, Lemma 11].

We can extend this result to give the following theorem.

Theorem 9. For some arbitrarily chosen state $\hat{x}$, let $\hat{\tau}=\inf \left\{t: X_{t}=\hat{x}\right\}$. Then, for any $\varepsilon>0$, there exists a $\beta>0$ depending only on $A$ and $\gamma$ such that, for any measure $\mathbb{Q} \in \mathcal{Q}_{\gamma}$,

$$
\mathbb{E}^{\mathbb{Q}}\left[\mathrm{e}^{\beta(\hat{\imath}-t)^{+}} \mid \mathcal{F}_{t}\right] \leq(1+\varepsilon)
$$

for any $t$.

Proof. Define the BSDE driver

$$
g_{\gamma}(x, z):=\sup _{B: B \sim_{\gamma} A}\left(z^{\top}(B-A) x\right) .
$$

By Lemma 2 and Remark 2, we can see that $g_{\gamma}$ is $\gamma$-balanced. By Lemma 1, $g_{\gamma}$ is Lipschitz with respect to the $\|\cdot\|_{M}$ seminorm. 
Let $f(\omega, t, z)=z^{\top}\left(\lambda_{t}-A X_{t-}\right)$, where $\lambda$ is a vector process generating $\mathbb{Q} \in \mathcal{Q}_{\gamma}$, in the sense of Definition 4. For any $T>0$, we can consider the BSDEs with terminal value $\mathrm{e}^{\beta(\hat{\tau} \wedge T)}$ at time $T$, and drivers $g_{\gamma}$ and $f$. Let $Y^{g}$ and $Y^{f}$ denote the corresponding solutions, for which direct calculation shows that

$$
Y_{t}^{g}=\sup _{B \sim \gamma A} \mathbb{E}^{B}\left[\mathrm{e}^{\beta(\hat{\tau} \wedge T)} \mid \mathcal{F}_{t}\right], \quad Y_{t}^{f}=\mathbb{E}^{\mathbb{Q}}\left[\mathrm{e}^{\beta(\hat{\tau} \wedge T)} \mid \mathcal{F}_{t}\right] .
$$

We can see that $f$ is $\gamma$-balanced and, furthermore, that $g_{\gamma}\left(X_{t-}, z\right) \geq f(\omega, t, z)$. By the finite-time comparison theorem (Theorem 2) we see that $Y_{t}^{g} \geq Y_{t}^{f}$ for all $t$. Hence,

$$
\sup _{B \sim_{\gamma} A} \mathbb{E}^{B}\left[\mathrm{e}^{\beta(\hat{\tau} \wedge T)} \mid \mathcal{F}_{t}\right] \geq \mathbb{E}^{\mathbb{Q}}\left[\mathrm{e}^{\beta(\hat{\tau} \wedge T)} \mid \mathcal{F}_{t}\right]
$$

and, taking $T \rightarrow \infty$, as $\hat{\tau}$ is a.s. finite, by the monotone convergence theorem we see that

$$
\mathbb{E}^{\mathbb{Q}}\left[\mathrm{e}^{\beta \hat{\tau}} \mid \mathcal{F}_{t}\right] \leq \sup _{B \sim \sim^{A}} \mathbb{E}^{B}\left[\mathrm{e}^{\beta \hat{\tau}} \mid \mathcal{F}_{t}\right] .
$$

Now, under each rate matrix $B$,

$$
\mathbb{E}^{B}\left[\mathrm{e}^{\beta \hat{\tau}} \mid \mathcal{F}_{t}\right]=\mathbb{E}^{B}\left[\mathrm{e}^{\beta\left(t \wedge \hat{\tau}+(\hat{\tau}-t)^{+}\right)} \mid \mathcal{F}_{t}\right] \leq \mathrm{e}^{\beta(t \wedge \hat{\tau})} \mathbb{E}^{B}\left[\mathrm{e}^{\beta(\hat{\tau}-t)^{+}} \mid \mathcal{F}_{t}\right] .
$$

For every $x \in \mathcal{X}$, by the Markov property and Theorem 8 , we have

$$
\mathbb{E}^{B}\left[\mathrm{e}^{\beta(\hat{\imath}-t)^{+}} \mid X_{t}=x\right]=\mathbb{E}^{B}\left[\mathrm{e}^{\beta \hat{\tau}} \mid X_{0}=x\right] \leq 1+\varepsilon .
$$

Hence,

$$
\mathbb{E}^{\mathbb{Q}}\left[\mathrm{e}^{\beta \hat{\tau}} \mid \mathcal{F}_{t}\right]=\mathbb{E}^{\mathbb{Q}}\left[\mathrm{e}^{\beta \hat{\tau}} \mid X_{t}\right] \leq \mathrm{e}^{\beta(t \wedge \hat{\tau})}(1+\varepsilon),
$$

and rearrangement yields the result.

Corollary 1. Let $\hat{\tau}$ be the first hitting time of a state $\hat{x}$. Then, for any $\beta \geq 0$ and any $\mathbb{Q} \in Q_{\gamma}$, there exists a constant $k$ such that

$$
\mathbb{E}^{\mathbb{Q}}\left[(1+\hat{\tau})^{1+\beta} \mid \mathcal{F}_{t}\right] \leq k(1+t)^{1+\beta} .
$$

Proof. From Theorem 9 we know that, for all $\gamma$ sufficiently small, $\mathbb{E}^{\mathbb{Q}}\left[\mathrm{e}^{\gamma(\hat{\tau}-t)^{+}} \mid \mathcal{F}_{t}\right] \leq$ $1+\varepsilon$. Hence, we can see that for any $\beta>0$ there exists some constant $k$ such that

$$
\mathbb{E}^{\mathbb{Q}}\left[\left(1+(\hat{\tau}-t)^{+}\right)^{1+\beta} \mid \mathcal{F}_{t}\right] \leq k
$$

As $t \geq 0$, we have the bound

$$
\begin{aligned}
(1+\hat{\tau})^{1+\beta} & \leq(1+t)^{1+\beta}\left(\frac{1+t+(\hat{\tau}-t)^{+}}{1+t}\right)^{1+\beta} \\
& \leq(1+t)^{1+\beta}\left(1+(\hat{\tau}-t)^{+}\right)^{1+\beta}
\end{aligned}
$$

The result follows.

\section{Applications}

We now present some novel applications of these methods. We begin with the archetypal Markovian control problem. A related approach for ergodic control problems was considered in [7]. A general setting for control of a marked point process to deterministic times was given in [10]. 


\subsection{Control to a stopping time}

Consider the problem of minimizing a cost

$$
Y_{t}=\underset{u}{\operatorname{essinf}} \mathbb{E}^{u}\left[\int_{] t, \tau]} L\left(s, Y_{s-}, X_{s-}, u_{s}\right) \mathrm{d} s+\phi\left(\tau, X_{\tau}\right) \mid \mathcal{F}_{t}\right],
$$

where

- $U$ is a space of controls, which is a separable metric space,

- $u$ is a $U$-valued predictable process,

- $\phi$ is a terminal cost function with $\phi(t, x) \leq c\left(1+t^{\beta}\right)$ for some $\beta>0$,

- $L: \mathbb{R}^{+} \times \mathbb{R} \times \mathcal{X} \times U \rightarrow \mathbb{R}$ is a measurable cost function, with

$$
|L(t, y, x, u)| \leq c\left(1+t^{\hat{\beta}}\right), \quad \frac{L(t, y, x, u)-L\left(t, y^{\prime}, x, u\right)}{y-y^{\prime}} \in[-c, 0],
$$

for some $c \in \mathbb{R}^{+}$and some $\hat{\beta}<\beta$,

- $\mathbb{E}^{u}$ is the expectation under which at time $t$, for the path $\omega, X$ jumps from state $e_{i}$ to state $e_{j}$ at a rate $e_{j}^{\top} A^{u_{t}(\omega)} e_{i}$, for some measurable matrix valued function $A^{(\cdot)}: U \rightarrow$ \{rate matrices\},

- for some $\gamma>0$, for all $u \in U$, the matrices $A^{u} \sim_{\gamma} A$, for some reference rate matrix $A$ under which $X$ is a uniformly ergodic Markov chain,

- $\tau$ is the first hitting time of a collection of points in $X$.

We shall write $\mathbb{E}$ for the expectation under which $X$ has rate matrix $A$.

We define the Hamiltonian

$$
f(x, t, y, z)=\inf _{v \in U}\left\{L(t, y, x, v)+z^{\top}\left(A^{v}-A\right) x\right\} .
$$

As $A^{u} \sim_{\gamma} A$, by Lemma 2 we see that $f$ is $\gamma$-balanced and the requirements of Theorem 3 are satisfied. Therefore, the BSDE (1) with driver $f$ admits a unique solution with bounded growth. By Theorem 6, the solution to the BSDE is Markovian, that is, $Y_{t}=u\left(t, X_{t}\right)$ and $e_{i}^{\top} Z_{t}=u\left(t, e_{i}\right)$, for some function $u$.

If the infimum in (4) is attained, then there exists (assuming the continuum hypothesis in [17]) a measurable function $\kappa: \mathcal{X} \times \mathbb{R}^{+} \times \mathbb{R} \times \mathbb{R}^{N} \rightarrow U$ such that

$$
f(x, t, y, z)=L(t, y, x, \kappa(x, t, y, z))+z^{\top}\left(A^{\kappa(x, t, y, z)}-A\right) x .
$$

We then have the following theorem.

Theorem 10. In the setting described above, let $(Y, Z)$ be the solution to the BSDE (1) with driver $f$. Then the following statements hold.

(i) For an arbitrary control $u$, if

$$
Y_{t}^{u}=\mathbb{E}_{x}^{u}\left[\int_{] t, \tau]} L\left(s, Y_{s-}^{u}, X_{s-}, u_{s}\right) \mathrm{d} s+\phi\left(\tau, X_{\tau}\right) \mid \mathcal{F}_{t}\right]
$$

then $Y_{t}^{u} \geq Y_{t}$, and equality holds if and only if

$$
L\left(t, Y_{t-}, X_{t-}, u_{t}\right)+\left(Z_{t}\right)^{\top}\left(A^{u_{t}}-A\right) X_{t-}=f\left(X_{t-}, t, Y_{t-}, Z_{t}\right), \quad \mathrm{d} \mathbb{P} \times \mathrm{d} t \text {-a.e. }
$$


(ii) If the infimum is attained in (4), then the control $u_{t}^{*}=\kappa\left(X_{t-}, t, Y_{t-}, Z_{t}\right)$ verifies $Y_{t}^{u^{*}}=Y_{t}$, and is an optimal feedback control.

Proof. We see that $Y_{\tau}=Y_{\tau}^{u}=\phi\left(\tau, X_{\tau}\right)$. By definition of the Hamiltonian, for an arbitrary control $u$ we have

$$
f(x, t, y, z) \leq L\left(t, y, x, u_{t}\right)+z^{\top}\left(A^{u_{t}}-A\right) x,
$$

and so by the comparison theorem (Theorem 5), as $Y^{u}$ solves the BSDE with the right-hand side of this inequality as a driver, we see that $Y_{t}^{u} \geq Y_{t}$ for every $t$, and the desired condition for equality holds. Statement (ii) also holds by an application of the comparison theorem.

\subsection{Stochastic shortest paths}

Let $g$ be a directed graph, represented by nodes in $\mathcal{X}$. Let the distance from node $e_{i}$ to $e_{j}$ be given by $e_{j}^{\top} D e_{i}$ for some matrix $D$. Let $A$ be the transition matrix of a continuous time random walk on this graph, so that $e_{j}^{\top} A e_{i}=\left(1 /\left(e_{i}^{\top} D e_{j}\right)\right) /\left(\sum_{j} 1 /\left(e_{i}^{\top} D e_{j}\right)\right)$ for $i \neq j$, and $e_{i}^{\top} A e_{i}=-\sum_{j \neq i} e_{j}^{\top} A e_{i}$. The expected time to reach a node $x$ starting from a node $x^{\prime}$ is then given by $\mathbb{E}\left[\tau \mid X_{0}=x^{\prime}\right]$, where $\tau$ is the first hitting time of $x$.

Now, suppose that it is possible to choose to 'walk faster' on those paths which are heading in the 'right' direction. This could be modelled by a change of measure, where the transition matrix $A$ is replaced by a matrix $A^{u}$, where $u$ is some control. Provided that $A^{u} \sim_{\gamma} A$ for some $\gamma$, as shown in Section 4.1, the expected time to hitting $x$ under the optimal control is then the solution of the BSDE to $\tau$ with driver $f(x, z)=\inf _{u}\left\{z^{\top}\left(A^{u}-A\right) x\right\}$ and terminal value $\xi=\tau$.

Alternatively, we can consider the expected remaining time to hitting $x$ under the optimal control as the solution of the BSDE to $\tau$ with driver $f^{\prime}(x, z)=\inf _{u}\left\{z^{\top}\left(A^{u}-A\right) x\right\}+1$, and terminal value $\xi^{\prime}=0$. If the earlier BSDE has solution $(Y, Z)$, and this variant has solution $\left(Y^{\prime}, Z^{\prime}\right)$, then we can see that $Y_{t}=Y_{t}^{\prime}+t$, but that $Y_{t}^{\prime}$ satisfies the requirements of Theorem 7 , and therefore its solution is of the form $Y_{t}^{\prime}=u\left(X_{t}\right)$ for some $u: \mathcal{X} \rightarrow \mathbb{R}$, which may be convenient for calculation.

\subsection{Reliability for networks with control}

Consider a model for transmission of messages over a finite network. A message is to be transmitted from a node $x_{0}$ to a node $x_{1}$, and each node $e_{i}$ naturally passes messages to node $e_{j}$ at a rate $e_{j}^{\top} A e_{i}$. We wish to examine the probability that the message eventually reaches its destination. When the network is flawless this probability is always 1 . However, suppose that each node $x$ loses the message at a rate $r_{x}$, and that there is a (possibly empty) collection of nodes $\Xi$ at which a message is irretrievably lost. Without any control, we can consider this as a linear BSDE to a stopping time.

Consider the Markov chain describing the motion of a message. Let $\tau$ be the first hitting time of $\left\{x_{1}\right\} \cup \Xi$, and let $Y_{t}$ be the solution to the BSDE

$$
I_{\left\{X_{\tau}=x_{1}\right\}}=Y_{t}-\int_{] t, \tau]}-r_{X_{t-}} Y_{u-} \mathrm{d} u+\int_{] t, \tau]} Z_{u}^{\top} \mathrm{d} M_{u},
$$

so that

$$
Y_{t}=\mathbb{E}\left[\exp \left(-\int_{] t, \tau]} r_{X_{s-}} \mathrm{d} s\right) I_{\left\{X_{\tau}=x_{1}\right\}} \mid \mathcal{F}_{t}\right] .
$$

Now, suppose that we can extend this model, so that each node has some control over where a message is sent. Each node has a control $u$ with which it can modify transitions so that they 
occur at a rate $e_{i}^{\top} A^{u} e_{j}$ for some $A^{u} \sim_{\gamma} A$, so as to maximise the probability that the message reaches its target. The maximal probability is then given by solving the BSDE

$$
I_{\left\{X_{\tau}=x_{1}\right\}}=Y_{t}-\int_{] t, \tau]}-r_{X_{s-}} Y_{s-}+\sup _{u}\left\{Z_{s}^{\top}\left(A^{u}-A\right) X_{s-}\right\} \mathrm{d} s+\int_{] t, \tau]} Z_{u}^{\top} \mathrm{d} M_{u},
$$

and the optimal policy for the active node $X_{t-}$ at time $t$ is given by

$$
u^{*}=\underset{u}{\arg \max }\left\{Z_{t}^{\top}\left(A^{u}-A\right) X_{t-}\right\}
$$

\subsection{Non-Ohmic Electronic circuits}

We now give a different situation, where we consider an electronic circuit. The theory of circuits of resistors is described using Kirchoff's laws and Ohm's law, a more detailed presentation of the approach we take here can be found in [16, Chapter 1].

Consider a circuit of resistors. Let the circuit be described by a graph represented in $\mathcal{X}$, and let the edge $\left(e_{i}, e_{j}\right)$ have resistance $R_{i, j}$. Then it follows from Ohm's law and Kirchoff's laws that, on a source set $\Xi$, the voltage potential is a prescribed function $\phi$, and off the source set the voltage potential $v$ in the circuit is a harmonic function. In particular, if $w_{i, j}=1 / R_{i, j}$ denotes the conductance over the edge $\left(e_{i}, e_{j}\right)$, we have

$$
\begin{cases}v\left(e_{i}\right)=\phi\left(e_{i}\right), & e_{i} \in \Xi \\ v\left(e_{i}\right) \sum_{j} w_{i, j}=\sum_{j} w_{i, j} v\left(e_{j}\right), & e_{i} \notin \Xi\end{cases}
$$

Let $A$ be the matrix defined by $e_{j}^{\top} A e_{i}=w_{i, j}$ for $i \neq j$ and $e_{i}^{\top} A e_{i}=-\sum_{j} w_{i, j}$. Then we consider a Markov chain $X$ with rate matrix $A$, and can show that

$$
v\left(e_{i}\right)=\mathbb{E}^{A}\left[\phi\left(X_{\tau}\right) \mid X_{0}=e_{i}\right]
$$

where $\tau$ is the first hitting time of the source set.

Now suppose that our circuit no longer consists purely of resistors, but that it also has diodes. These ubiquitous electronic components fail to satisfy Ohm's law, and so the above representation in terms of a Markov chain fails. However, it is possible to write down a nonlinear relationship between voltage, current, and resistance which is satisfied (see, for example, [11, Section 5.2]). In particular, if we know the voltage drop over an edge $V_{i, j}=v\left(e_{i}\right)-v\left(e_{j}\right)$ we can write $I_{i, j} R_{i, j}\left(V_{i, j}\right)=V_{i, j}$, where $I_{i, j}$ is the current passing over the edge. From [11], the relation for an $n p$-type diode, for example, is given by

$$
I_{i . j}=I^{s}\left(\exp \left(\frac{V_{i, j}}{V^{T}}\right)-1\right)
$$

where $I^{S}$ and $V^{T}$ are constants based on the properties of the diode. Rearranging this gives the implied resistance

$$
R_{i, j}\left(V_{i, j}\right)=\frac{V_{i, j}}{I^{s}\left(\exp \left(V_{i, j} / V^{T}\right)-1\right)}>0,
$$

which is a Lipschitz function of $V_{i, j}$, and is bounded away from zero (and $\infty$ ) over any finite interval.

Writing $\boldsymbol{v}$ for the vector with entries $e_{i}^{\top} \boldsymbol{v}=v\left(e_{i}\right)$, and $w_{i, j}(\boldsymbol{v})=\left(R_{i, j}\left(V_{i j}\right)\right)^{-1}$ for the implied conductance, we can construct a matrix $A^{v}$ with $e_{j}^{\top} A^{v} e_{i}=w_{i, j}(\boldsymbol{v})$ for $i \neq j$ and 
$e_{i}^{\top} A^{v} e_{i}=-\sum_{j} w_{i, j}(\boldsymbol{v})$. For a given voltage potential vector $\boldsymbol{v}$, this $A^{\boldsymbol{v}}$ is the transition matrix based on the implied conductances at the potential $\boldsymbol{v}$.

Let $A$ be as before, now defined by replacing each diode with a resistor with resistances $\tilde{R}_{i, j}$. As the circuit is finite, $A$ generates a uniformly ergodic Markov chain.

We then consider the BSDE

$$
\phi\left(X_{\tau}\right)=Y_{t}-\int_{] t, \tau]} Z_{s}^{\top}\left(A^{Z_{s}}-A\right) X_{s-} \mathrm{d} s+\int_{] t, \tau]} Z_{t}^{\top} \mathrm{d} M_{t} .
$$

This is a time homogeneous Markovian BSDE to a first hitting time. The terminal value $\phi\left(X_{\tau}\right)$ is bounded and we can directly verify that the driver $f\left(X_{s-}, Z_{s}\right)=Z_{s}^{\top}\left(A^{Z_{s}}-A\right) X_{s-}$ is locally $\gamma$-balanced, and $f(x, 0)=0$. By Theorem 4 , this BSDE admits a unique bounded solution, and by Theorem 7 it is of the form $Y_{t}=v\left(X_{t}\right)$, which is precisely the voltage potential on the circuit with a diode.

\section{Conclusions}

We have considered BSDEs on Markov chains when the terminal time is replaced by a nonbounded stopping time, and no strict monotonicity property in $Y$ (i.e. a 'discounting' term) is given for $f$. We have shown that, given appropriate polynomial growth bounds on the terminal value and the driver, and given sufficient integrability of the stopping time, these equations admit unique solutions with uniformly controlled growth in time. For the special case of first hitting times of a set, we have seen that the integrability conditions on the stopping time are indeed satisfied, and so these BSDEs admit unique solutions.

We have then considered various applications to these equations; in particular, to control problems which terminate at a hitting time, and to problems of nonlinear behaviour on certain graphs, for example to network reliability. We have also seen how this gives a stochastic approach to our understanding of electronic circuits without Ohm's law.

We can also extend these approaches to more general types of BSDEs, either to the classic Brownian setting of [19], or to allow for Poisson jumps as in [22]. For the Brownian case, the required ergodicity properties may be derivable from [13]; however, such an extension is nontrivial. On the other hand, if $\tau$ is restricted to be the first exit time of a set, then standard estimates may be available to give the required integrability, in which case our results will extend directly.

\section{Acknowledgements}

I would like to thank Shige Peng, Gechun Liang, Jeff Dewynne, and Chris Lustri for useful conversations; in particular, to Chris Lustri for discussions on circuits violating Ohm's law. I would also like to thank the Oxford-Man Institute for Quantitative Finance for research support.

\section{References}

[1] Bismut, J.-M. (1973). Conjugate convex functions in optimal stochastic control. J. Math. Anal. Appl. 44, $384-404$.

[2] BRIAND, P. AND Hu, Y. (1998). Stability of BSDEs with random terminal time and homogenization of semilinear elliptic PDEs. J. Funct. Anal. 155, 455-494.

[3] CoHen, S. N. (2012). Representing filtration consistent nonlinear expectations as g-expectations in general probability spaces. Stoch. Process. Appl. 122, 1601-1626.

[4] Cohen, S. N. ANd Elliott, R. J. (2008). Solutions of backward stochastic differential equations on Markov chains. Commun. Stoch. Anal. 2, 251-262. 
[5] Cohen, S. N. and Elliott, R. J. (2010). Comparisons for backward stochastic differential equations on Markov chains and related no-arbitrage conditions. Ann. Appl. Prob. 20, 267-311.

[6] Cohen, S. N. And Elliott, R. J. (2012). Existence, uniqueness and comparisons for BSDEs in general spaces. Ann. Prob. 40, 2264-2297.

[7] Cohen, S. N. And Hu, Y. (2013). Ergodic BSDEs driven by Markov chains. SIAM J. Control Optimization 51, 4138-4168.

[8] Cohen, S. N. And SzPruch, L. (2012). On Markovian solutions to Markov chain BSDEs. Numer. Algebra Control Optimization 2, 257-269.

[9] Cohen, S. N., Elliott, R. J. and Pearce, C. E. M. (2010). A general comparison theorem for backward stochastic differential equations. Adv. Appl. Prob. 42, 878-898.

[10] Confortola, F. And Fuhrman, M. (2013). Backward stochastic differential equations and optimal control of marked point processes. SIAM J. Control Optimization 51, 3592-3623.

[11] Crecraft, D. I. and Gergely, S. (2002). Analog Electronics: Circuits, Systems and Signal Processing. Butterworth-Heinemann, Oxford.

[12] Darling, R. W. R. And Pardoux, E. (1997). Backwards SDE with random terminal time and applications to semilinear elliptic PDE. Ann. Prob. 25, 1135-1159.

[13] Debussche, A., Hu, Y. And Tessitore, G. (2011). Ergodic BSDEs under weak dissipative assumptions. Stoch. Process. Appl. 121, 407-426.

[14] Elliott, R. J., Aggoun, L. And Moore, J. B. (1995). Hidden Markov Models. Estimation and Control. Springer, New York.

[15] Föllmer, H. And Schied, A. (2002). Stochastic Finance. An Introduction in Discrete Time (De Gruyter Studies Math. 27). De Gruyter, Berlin.

[16] Grimmett, G. (2010). Probability on Graphs. Cambridge University Press.

[17] McShane, E. J. And Warfield, R. B., JR. (1967). On Filippov's implicit functions lemma. Proc. Amer. Math. Soc. 18, 41-47.

[18] Meyn, S. And Tweedie, R. L. (2009). Markov Chains and Stochastic Stability, 2nd edn. Cambridge University Press.

[19] Pardoux, E. And Peng, S. G. (1990). Adapted solution of a backward stochastic differential equation. Systems Control Lett. 14, 55-61.

[20] Rogers, L. C. G. And Williams, D. (2000). Diffusions, Markov Processes, and Martingales, Vol. 1, Foundations, 2nd edn. Cambridge University Press.

[21] Royer, M. (2004). BSDEs with a random terminal time driven by a monotone generator and their links with PDEs. Stoch. Stoch. Reports 76, 281-307.

[22] Royer, M. (2006). Backward stochastic differential equations with jumps and related non-linear expectations. Stoch. Process. Appl. 116, 1358-1376. 